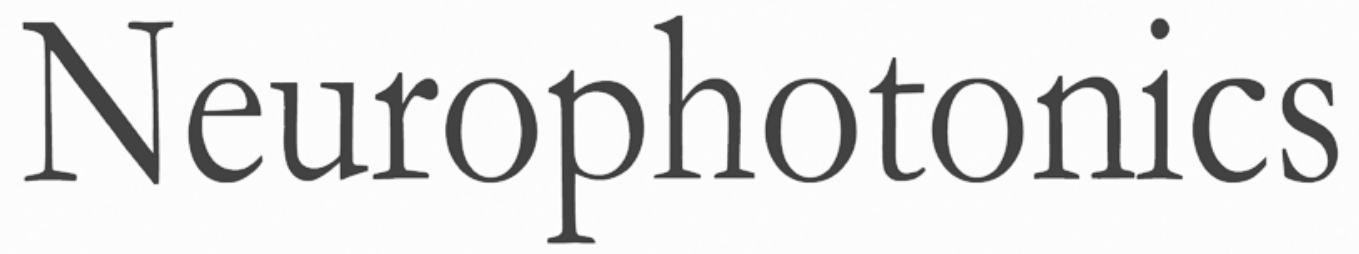

\title{
Functional near-infrared spectroscopy for intraoperative brain mapping
}

Tianming Qiu

N. U. Farrukh Hameed

Yuerong Peng

Shuheng Wang

Jinsong $\mathrm{Wu}$

Liangfu Zhou 


\title{
Functional near-infrared spectroscopy for intraoperative brain mapping
}

\author{
Tianming Qiu, ${ }^{a, \dagger}$ N. U. Farrukh Hameed, ${ }^{a, \dagger}$ Yuerong Peng, ${ }^{b}$ Shuheng Wang, ${ }^{c}$ \\ Jinsong $\mathrm{Wu}^{\mathrm{a}, *}$ and Liangfu Zhou ${ }^{\mathrm{d}, *}$ \\ ${ }^{a} F u d a n$ University, Huashan Hospital, Glioma Surgery Division, Department of Neurosurgery, Shanghai, China \\ ${ }^{b}$ Fudan University, Huashan Hospital, Department of Anesthesia, Shanghai, China \\ 'Yale University, Statistics and Data Science Department, Connecticut, United States \\ ${ }^{\mathrm{d} F u d a n}$ University, Huashan Hospital, Department of Neurosurgery, Shanghai, China
}

\begin{abstract}
Functional near-infrared spectroscopy (fNIRS) is a relatively new seizure-free technique and its value for intraoperative brain mapping is unknown. We examine the feasibility of fNIRS for intraoperative functional brain mapping. A $1 \times 1 \mathrm{~cm}^{2}$ density fNIRS probe specially designed for intraoperative use was used to map brain function in adult patients undergoing awake brain surgery and performing motor and/or language tasks. The ability of fNIRS for functional mapping was compared with direct cortical stimulation (DCS) and regression was used to determine if mean blood pressure (MBP) and blood hemoglobin influenced fNIRS measurements. Eighteen patients underwent awake craniotomy and performed 19 language- and 17 motor-related tasks. fNIRS mapping was highly correlated with DCS for 10 language- and 7 motor-related tasks. fNIRS was able to detect functional language $(p<0.001)$ and motor areas $(p=0.002)$. Compared to DCS, $\mathrm{fNIRS}$ was less accurate in determining both functional language (at least $22.64 \%, p<0.001$ ) and motor areas (at least $32.74 \%, p<0.001$ ). Higher MBP and blood hemoglobin were associated with better fNIRS results $(p=0.045$ and 0.007 , respectively). No seizures or other complications occurred during fNIRS measurement. fNIRS is a promising seizurefree technique for intraoperative brain mapping. The accuracy of current technology needs further development for clinical use. $\odot$ The Authors. Published by SPIE under a Creative Commons Attribution 4.0 Unported License. Distribution or reproduction of this work in whole or in part requires full attribution of the original publication, including its DOI. [DOI: 10.1117/1.NPh.6.4.045010]

Keywords: brain mapping; functional; infrared; spectroscopy; cortex.
\end{abstract}

Paper 19083R received Aug. 24, 2019; accepted for publication Nov. 5, 2019; published online Nov. 25, 2019.

\section{Introduction}

Functional brain imaging is an area of extensive ongoing research, and with the rapid advancement in technology and subtle applications of artificial intelligence, this field has evolved very rapidly in the past decade. This is extremely beneficial for neuroscientists and neurosurgeons in particular, because the preoperative and intraoperative functional mapping of brain regions can completely alter the surgical outcomes and prognoses of patients.

Functional brain imaging techniques can be broadly grouped into electrophysiological and metabolic techniques. Electrophysiological techniques, such as direct cortical electrical stimulation (DCS) and electroencephalogram (EEG), from the imaging perspective, better reflect neural activity. They can also promote therapeutic neuroplasticity by modifying neural circuits and have prolonged effect, which explains their therapeutic value in stroke patients. ${ }^{1-4}$ By comparison, metabolic techniques, such as functional magnetic resonance imaging (fMRI), photoacoustic imaging, and functional near-infrared spectroscopy (fNIRS), are less invasive and safer. ${ }^{5}$ They require a modest skillset for operation and have good spatial resolution. ${ }^{6}$ However, traditional functional imaging techniques have their weaknesses. EEG has a low spatial resolution and DCS can trigger intraoperative epileptic seizures in up to $5 \%$ of patients ${ }^{7}$ and cause patient discomfort during current alteration. ${ }^{8}$ Similarly, metabolic brain mapping

*Address all correspondence to Jinsong Wu, E-mail: wjsongc@126.com; Liangfu Zhou, E-mail: Ifzhouc@126.com

'Both authors contributed equally to this study. techniques generally have poor temporal resolution limiting their application for complex functional brain studies. ${ }^{6}$ The loud noise of fMRI can also influence interpretation of results. ${ }^{9}$ These disadvantages call for exploration of alternative options.

fNIRS is a unique technique that has the potential for functional cortical mapping. ${ }^{10,11}$ fNIRS measures changes in brain function by utilizing the different light absorption spectra of oxyhemoglobin $\left(\mathrm{O}_{2} \mathrm{Hb}\right)$ and deoxyhemoglobin $(\mathrm{HHb})$ within the near-infrared spectrum to interrogate the concentration of these substances in cerebral blood vessels. ${ }^{9}$ It is based on the principle that $\mathrm{O}_{2} \mathrm{Hb}$ and $\mathrm{HHb}$ (the main chromophores in the cortex) have an absorptive effect on light spectra and that hemodynamic variations reflected by spectral changes indicate brain activity. ${ }^{12}$ In recent years, fNIRS has advanced significantly and the common scalp probe has evolved into a relatively smaller and higher spatial resolution probe. ${ }^{13}$

While fNIRS has been applied intraoperatively to study corticocortical activity in eloquent brain regions ${ }^{14}$ and stereotactic functional localization, ${ }^{11}$ its nonoperative applications continue to expand ${ }^{15-27}$ and its value in surgical brain mapping remains limited. The present study explores the feasibility of fNIRS for intraoperative functional brain mapping using an enhanced probe and DCS for validation.

\section{Methods}

\subsection{Patient Demographics}

All patients enrolled in the study were from Huashan Hospital and the study was reviewed and approved by the hospital's Institutional Review Board. The patients include nine men and 
nine women aged 23 to 72 years. Inclusion criteria were as follows: patients with (1) primary brain lesion undergoing first surgery; (2) tumors located adjacent to language or motor area; (3) no contraindications to intraoperative electrophysiological monitoring; and (4) preoperative muscle strength greater than grade 3 and normal language function.

\subsection{Awake Craniotomy and Intraoperative DCS}

A monitored anesthesia care approach was adopted for all patients, as described previously. ${ }^{28}$ Awake craniotomy, DCS was used to locate functional motor and language cortical areas and positive loci were recorded. During motor mapping, compound muscle action potentials were monitored and cortical areas corresponding to hand-motor movement were marked with sterile tags and defined as motor-related areas. Similarly, during language mapping, patients counted from 1 to 50, and when speech arrest occurred, the corresponding area was tagged. Speech arrest was distinguished carefully from dysarthria. If mouth or pharyngeal muscles contracted, the corresponding area was identified as a mouth-motor area. In this study, both speech-arrest and mouthmotor areas were defined as language-related areas.

\section{3 fNIRS Signal Stability and Data Acquisition}

To measure cortical $\mathrm{Hb}$ concentrations intraoperatively, we utilized a device consisting of four recording probes based on a previous design. ${ }^{13}$ In contrast to their design, our probes are $1 \mathrm{~cm}$ apart, with four channels and higher resolution. The probes have fixable retractors at their ends for attachment to the brain surface. The four channels were named $\mathrm{CH} 1, \mathrm{CH} 5, \mathrm{CH} 6$, and CH10. fNIRS was carried out with a $695 / 830 \mathrm{~nm}$ (fixed wavelength determined by device engineers to have the highest signal-to-noise ratio) spectrometer equipped to our monitoring device (ETG4000; HITACHI Medical, Japan). Emitting light intensity was adjusted to $0.3 \mathrm{~mW}(\sim 1 / 12$ th of scalp fNIRS).

Following confirmation of signal stability in all cases (during installation, device technician adjusts the laser power to determine the strength and auto gain function to stabilize), the probe was used to record signals from normal and lesioned brain regions of each subject. In some cases, stability was tested by placing the probe on the positive sites following brain mapping, including motor and/or language areas [Figs. 1(a)-1(d)].

Data from fNIRS were acquired by block design. $\mathrm{O}_{2} \mathrm{Hb}$, $\mathrm{HHb}$, and total $\mathrm{Hb}(\mathrm{tHb})$ concentration changes corresponding to brain activity during tasks were measured for each run, which consisted of a 45-s stimulation block followed by a 45 -s rest block. Each run was repeated three times. A 30-s waiting time to establish the baseline was allowed before commencing the three runs. When the probes were placed on the hand-motor areas, patients were required to clench the fist repeatedly as a task. For the mouth-motor or speech (language-related) areas, number counting was the task. Throughout the process of fNIRS data acquisition, patients' vital signs and data including oxygen
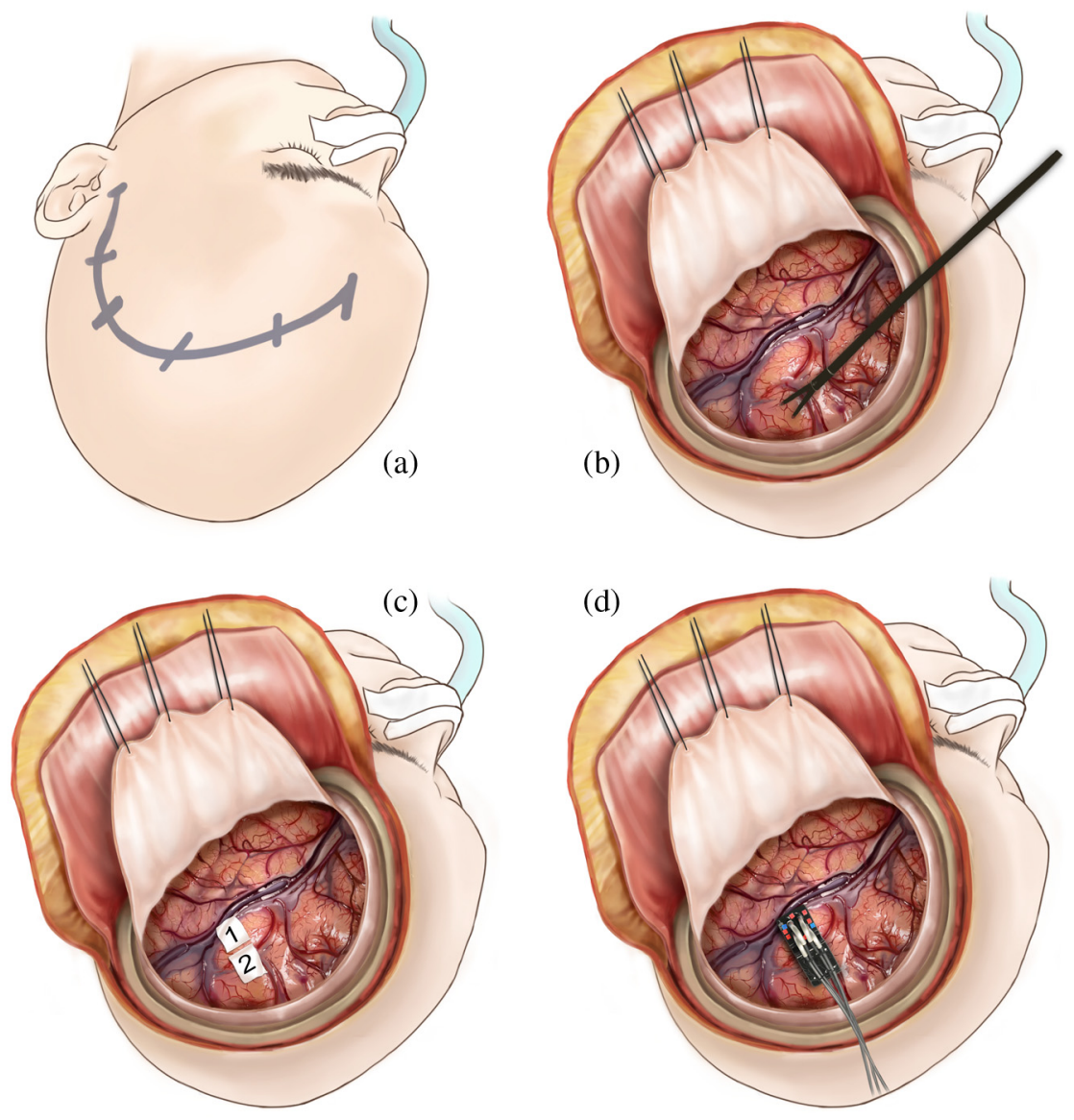

Fig. 1 Intraoperative brain mapping with functional near infrared spectroscopy: (a) following awake craniotomy, (b) DCS is performed to localize functional cortical areas, and (c) these regions are marked with sterile tags. (d) The improved four-channel fNIRS probe is then placed at these tagged regions to obtain readings while patients perform tasks. 
saturation and arterial $\mathrm{Hb}$ concentration were recorded (Hb concentrations were calculated using the Beer-Lambert law).

\subsection{Statistical Analysis}

The $t$-tests were performed to assess the performance of fNIRS in language and motor area localization. We also performed paired $t$-tests to compare the accuracies of fNIRS versus DCS in detecting language and motor function areas and determine statistical significance. Logistic regression was also performed to assess the effect of mean blood pressure (MBP) and blood $\mathrm{Hb}$ on fNIRS measurement. Statistical analysis was performed using SPSS version 19.0 for Windows (SPSS Inc., Chicago, Illinois).

\section{Results}

\subsection{Signal Stability}

Initially, the four channels yielded good signals, which were not stable. However, on repeated testing, all signals normalized, resolving the problem of signal instability. The adjustments that resulted in signal stabilization are as follows: the power is adjusted to the lowest level, the intensity of the near-infrared rays is reduced, and a fiber optic attenuator is added to the received signals to maintain stability. As shown in Fig. 2, these signals stabilized over time \{time course/ $[(\mathrm{mmol} / \mathrm{l}) \times \mathrm{mm}]\}$ on the user interface, with stable baselines for the different $\mathrm{Hb}$ parameters.
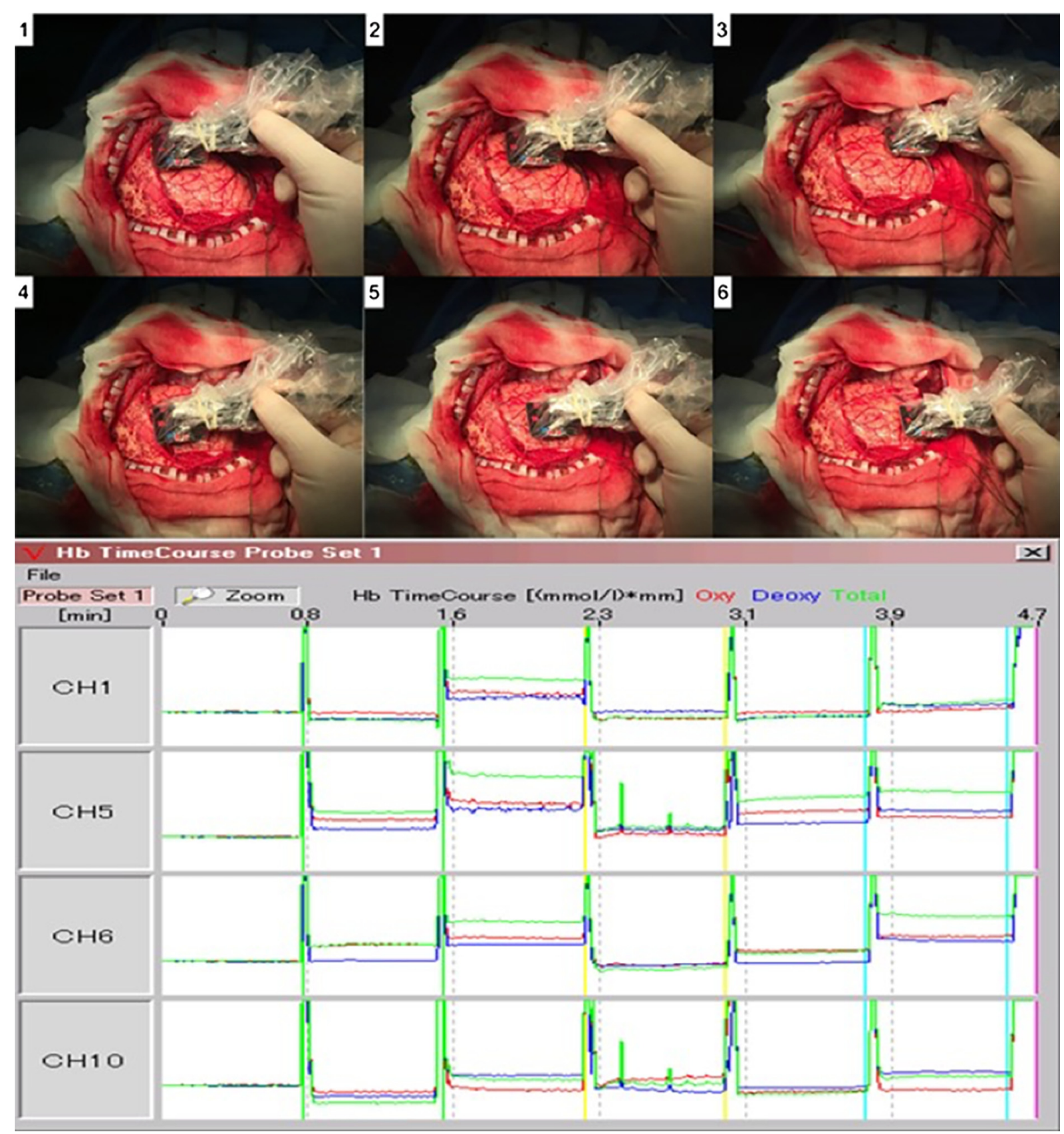

Fig. 2 Signal stability testing. The probe was used to record signals from normal and diseased brain regions of each subject. On repeated testing, the signals from all channels stabilized. 


\subsection{General Results}

Patients' vital signs and parameters including oxygen saturation and arterial $\mathrm{Hb}$ concentration were normal (Table 1). All patients underwent awake craniotomy and cooperated well with the intraoperatively administered hand-motor and language tasks. We acquired fNIRS signals from 36 DCS-determined sites and 17 motor- and 19 language-related positive sites.

\section{3 fNIRS Data Acquisition}

Through continuous mapping, we observed direct real-time information on the concentration of $\mathrm{Hb}$ in the vessels of the eloquent brain cortex. Of the administered tasks, 10 languagerelated and 7 hand-motor tasks yielded positive fNIRS results with curves highly correlating with block-designed tasks.

According to the signal acquisition schemes, as illustrated in Fig. 3, the following general trend of results was obtained for relative $\mathrm{O}_{2} \mathrm{Hb}, \mathrm{HHb}$, and $\mathrm{tHb}$ concentrations in the patients. When the probe was placed on the language area and the task sequence was implemented, a markedly elevated signal amplitude was detected by the fNIRS probe relative to the resting state. During the task phase (number counting) of each stimulation block, unique patterns of $\mathrm{Hb}$ curves in response to brain activation were observed. In several patients, only the $\mathrm{O}_{2} \mathrm{Hb}$ and

Table 1 Summary of intraoperative tasks performed by patients.

\begin{tabular}{|c|c|c|c|c|c|c|c|c|c|c|c|c|}
\hline Patient & Age & Sex & Pathology & MBP & $\begin{array}{l}\text { Hemoglobin } \\
(\mathrm{g} / \mathrm{L})\end{array}$ & $\begin{array}{l}\text { Oxygen } \\
\text { saturation } \\
(\%)\end{array}$ & $\begin{array}{c}\text { Functional } \\
\text { area } 1\end{array}$ & Task 1 & $\begin{array}{c}\text { Functional } \\
\text { area } 2\end{array}$ & Task 2 & $\begin{array}{c}\text { Functional } \\
\text { area } 3\end{array}$ & Task 3 \\
\hline 1 & 46 & $M$ & GBM (IV) & $168 / 95$ & 13.5 & 100 & LRT (P) & $\begin{array}{l}\text { Number } \\
\text { counting }\end{array}$ & $\operatorname{LRT}(\mathrm{P})$ & $\begin{array}{l}\text { Number } \\
\text { counting }\end{array}$ & LRT (P) & $\begin{array}{l}\text { Number } \\
\text { counting }\end{array}$ \\
\hline 2 & 29 & $F$ & A (II) & $175 / 82$ & 12.5 & 100 & MRT & Clenching fist & & & & \\
\hline 3 & 53 & $\mathrm{~F}$ & GBM (IV) & $195 / 83$ & 11.2 & 100 & LRT & $\begin{array}{l}\text { Number } \\
\text { counting }\end{array}$ & & & & \\
\hline 4 & 72 & M & GBM (IV) & $120 / 72$ & 9.7 & 100 & MRT & Clenching fist & & & & \\
\hline 5 & 38 & $M$ & A (II) & $175 / 78$ & 13 & 99 & LRT & $\begin{array}{l}\text { Number } \\
\text { counting }\end{array}$ & MRT (P) & Clenching fist & & \\
\hline 6 & 49 & $\mathrm{~F}$ & AA (III) & $148 / 82$ & 9.6 & 100 & MRT (P) & Clenching fist & LRT (P) & $\begin{array}{l}\text { Number } \\
\text { counting }\end{array}$ & & \\
\hline 7 & 48 & $M$ & $\mathrm{O}(\mathrm{II})$ & $166 / 76$ & 14.9 & 100 & MRT (P) & Clenching fist & MRT (P) & Clenching fist & LRT (P) & $\begin{array}{l}\text { Number } \\
\text { counting }\end{array}$ \\
\hline 8 & 34 & M & A (II) & $180 / 80$ & 14.4 & 97 & MRT (P) & Clenching fist & MRT (P) & Clenching fist & MRT (P) & Clenching fist \\
\hline 9 & 42 & $F$ & A (II) & $173 / 83$ & 8.1 & 100 & MRT & Clenching fist & MRT & Clenching fist & MRT & Clenching fist \\
\hline 10 & 43 & $\mathrm{~F}$ & $\mathrm{O}(\mathrm{II})$ & $142 / 77$ & 9.1 & 100 & MRT & Clenching fist & MRT & Clenching fist & LRT & $\begin{array}{l}\text { Number } \\
\text { counting }\end{array}$ \\
\hline 11 & 60 & M & $\mathrm{AO}$ (III) & $133 / 70$ & 12.8 & 100 & MRT & Clenching fist & & & & \\
\hline 12 & 29 & $F$ & A (II) & $146 / 73$ & 12.7 & 100 & LRT & $\begin{array}{l}\text { Number } \\
\text { counting }\end{array}$ & & & & \\
\hline 13 & 34 & M & A (II) & $144 / 85$ & 13.2 & 100 & LRT (P) & $\begin{array}{l}\text { Number } \\
\text { counting }\end{array}$ & & & & \\
\hline 14 & 46 & $M$ & A (II) & $184 / 87$ & 13.1 & 100 & LRT (P) & $\begin{array}{l}\text { Number } \\
\text { counting }\end{array}$ & LRT (P) & $\begin{array}{l}\text { Number } \\
\text { counting }\end{array}$ & & \\
\hline 15 & 42 & $M$ & GBM (IV) & $143 / 86$ & 15.1 & 100 & LRT (P) & $\begin{array}{l}\text { Number } \\
\text { counting }\end{array}$ & LRT (P) & $\begin{array}{l}\text { Number } \\
\text { counting }\end{array}$ & & \\
\hline 16 & 49 & $\mathrm{~F}$ & $\mathrm{O}(\mathrm{II})$ & $146 / 75$ & 12.8 & 99 & LRT & $\begin{array}{l}\text { Number } \\
\text { counting }\end{array}$ & LRT & $\begin{array}{l}\text { Number } \\
\text { counting }\end{array}$ & & \\
\hline 17 & 23 & $\mathrm{~F}$ & A (II) & $134 / 70$ & 13.2 & 100 & MRT & Clenching fist & MRT & Clenching fist & & \\
\hline 18 & 32 & $\mathrm{~F}$ & GBM (IV) & $154 / 87$ & 11.1 & 100 & LRT & $\begin{array}{l}\text { Number } \\
\text { counting }\end{array}$ & LRT & $\begin{array}{l}\text { Number } \\
\text { counting }\end{array}$ & LRT & $\begin{array}{l}\text { Number } \\
\text { counting }\end{array}$ \\
\hline
\end{tabular}

Note: A, astrocytoma; AA, anaplastic astrocytoma; AO, anaplastic oligodendroglioma; GBM, glioblastoma; LRT, language related task (number counting); MRT, motor-related task (fist clenching); P, positive fNIRS measurement; O, oligodendroglioma. 


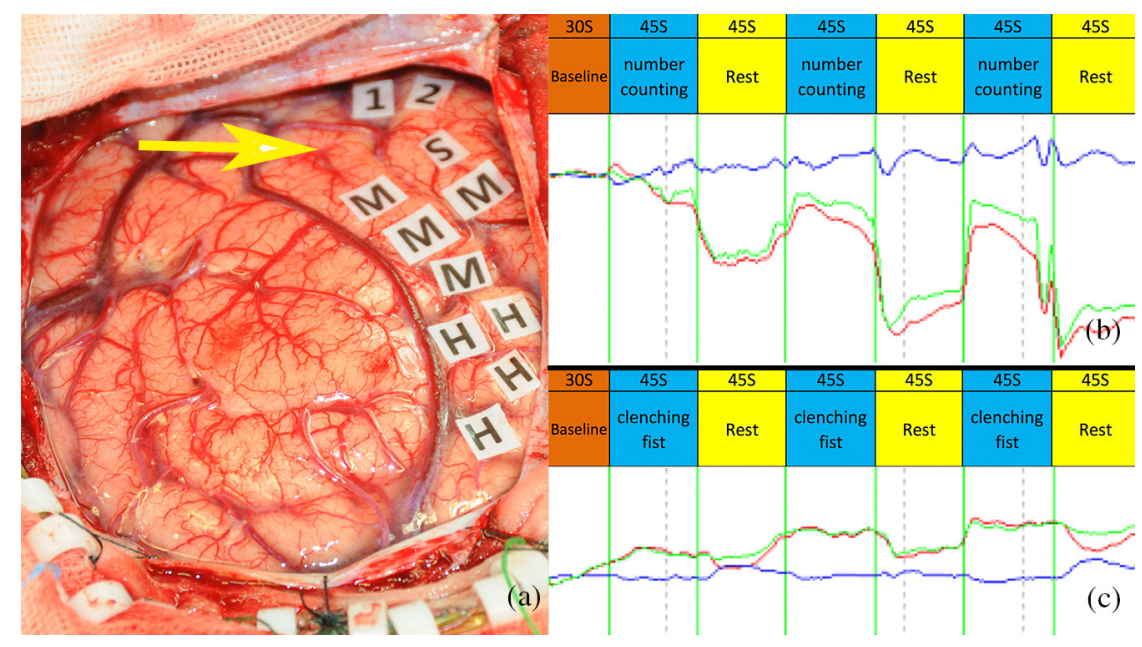

Fig. 3 Illustrated case 1. Following (a) awake craniotomy and DCS mapping of activated brain regions (arrow points in the anterior direction), (b) and (c) $\mathrm{O}_{2} \mathrm{Hb}$ (red) and total hemoglobin (green) curves rose significantly during (b) language and (c) hand-motor task blocks. However, the $\mathrm{HHb}$ curve (blue) trended at the baseline.

$\mathrm{tHb}$ curves rose noticeably above the baseline during tasks, while $\mathrm{HHb}$ trended around the baseline. At rest, the $\mathrm{O}_{2} \mathrm{Hb}$ curve fell back to the baseline.

By comparison, in some patients, $\mathrm{O}_{2} \mathrm{Hb}, \mathrm{HHb}$, and $\mathrm{tHb}$ all rose above the baseline during tasks. Similar to language sequences, when the probe was placed on the hand-motor area and when the task sequence (fist-clenching) was initiated, there was a significant elevation in the fNIRS signal amplitude. The $\mathrm{Hb}$ curves trended similarly to language tasks, but were relatively smoother than language curves, which had a spikier appearance. The elevations above baseline during languagerelated tasks were also much more pronounced than motorrelated tasks.

\subsection{Statistical Analysis}

One-sample $t$-tests showed that fNIRS was capable of detecting functional language $(p=0.00014)$ and motor areas $(p=$ $0.00205)$. Compared to DCS, fNIRS was less accurate in determining both functional language (at least $22.64 \%, p<0.001$ ) and motor areas (at least $32.74 \%, p<0.001$ ). On logistic regression, both blood $\mathrm{Hb}$ and $\mathrm{MBP}$ were positively correlated with positive fNIRS results (blood Hb coefficient $=0.76, p=0.007$ and MBP coefficient $=0.11, p=0.045$ ).

\section{Illustrative Cases}

1. The patient is a 48-year old male, who presented with a 1-month history of intermittent right hemifacial tingling and right lower limb numbness (Fig. 3). Preoperative structural and functional imaging confirmed a left hemispheric brain glioma that was resected by awake craniotomy. fNIRS probing of DCS-determined hand-motor $(H)$, mouth-motor $(M)$, and speech-arrest $(S)$ areas revealed a highly correlating pattern (in at least one channel) of $\mathrm{O}_{2} \mathrm{Hb}$ variation in response to brain activation. The $\mathrm{O}_{2} \mathrm{Hb}$ variation was similar to what is observed in most patients with positive results. This was particularly prominent during fist clenching and number counting tasks, where the $\mathrm{O}_{2} \mathrm{Hb}$ rose significantly during task periods and fell back to the baseline at rest. The HHb curve, on the other hand, correlated less prominently during these tasks and remained at baseline.

2. The second illustrated case is a 49-year old female, who presented with a 1-month history of seizures and normal bilateral muscle strength. MRI scans revealed a low-grade glioma in the right frontal and insular lobes, while fMRI scans showed that the motor and sensory cortices were located posterior to the lesion. Following awake craniotomy and DCS, the fNIRS probe was placed on brain regions corresponding to hand-motor $(H)$, mouth-motor $(M)$ regions, and hand-sensory $(S)$ areas. As the $\mathrm{O}_{2} \mathrm{Hb}$ curve rose during language and motor task periods, the $\mathrm{HHb}$ curve declined concomitantly [Fig. 4(a)]. In contrast to motor tasks, which displayed gentle $\mathrm{Hb}$ curves, the language task curves were bumpier and spikier [Fig. 4(b)]. In this case, during hand-motor stimulation blocks, the $\mathrm{O}_{2} \mathrm{Hb}$ curves continued to remain elevated above the baseline during rest following the task phase, whereas the $\mathrm{HHb}$ and $\mathrm{tHb}$ curves both declined similar to the general trend. However, in language mapping, the curves were not different from the general trend.

3. The third case is a 42-year old female, who presented with a 2-week history of sudden onset of seizures. MRI revealed a suspected low-grade left frontal lobe lesion. Following awake craniotomy, fNIRS probing of functional brain regions determined by DCS did not demonstrate significant activity of the $\mathrm{Hb}$ waves. Aside from a few exceptions, the $\mathrm{O}_{2} \mathrm{Hb}, \mathrm{HHb}$, and $\mathrm{tHb}$ curves all trended close to the baseline. Interestingly, integrated mapping analysis of hand-motor region probing still demonstrated an elevated $\mathrm{O}_{2} \mathrm{Hb}$ level with a concomitantly decreased $\mathrm{HHb}$ level. However, this was not seen for language mapping and no obvious trend was observed (Fig. 5). 


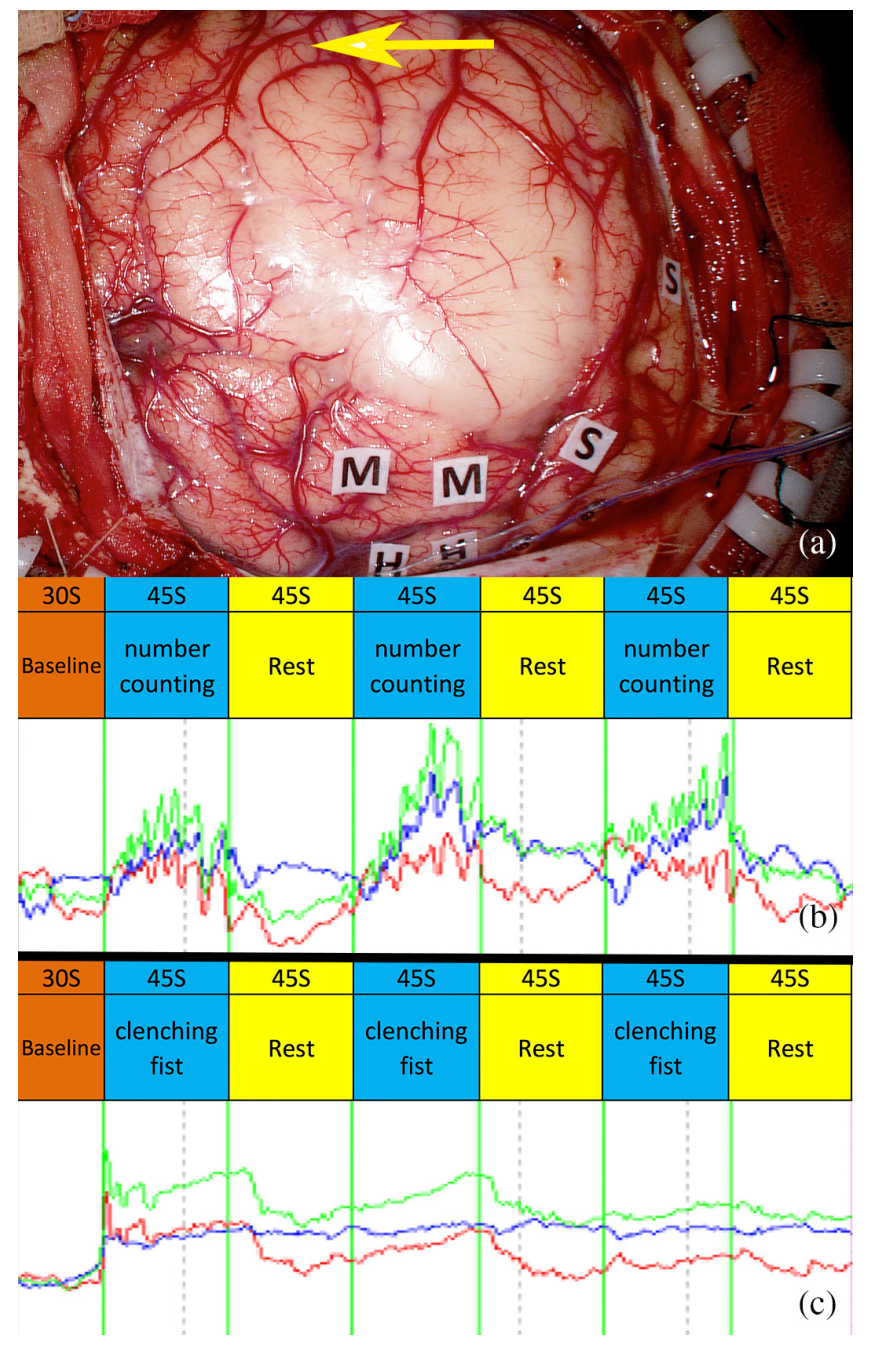

Fig. 4 Illustrated case 2. Following (a) awake craniotomy and DCS mapping of activated brain regions (arrow points in the anterior direction), (b) $\mathrm{O}_{2} \mathrm{Hb}$ (red), $\mathrm{HHb}$ (blue), and total hemoglobin (tHb) (green) curves all rose significantly during language tasks. These curves were also spikier compared to motor task curves. (c) For hand-motor tasks, while the $\mathrm{O}_{2} \mathrm{Hb}$ and $\mathrm{tHb}$ curves rose and fell in response to each task block, the $\mathrm{HHb}$ curve trended uniformly above the baseline following the initial task.

\section{Discussion}

In the present study, we demonstrated the feasibility of fNIRS for language and motor mapping during tumor surgery and validated our findings by comparing them with DCS results. fNIRS was used intraoperatively to locate eloquent areas in real-time by observing the dynamic changes in $\mathrm{Hb}$ concentration resulting from task activations. Moreover, factors associated with fNIRS accuracy were identified.

Past clinical studies have generally explored applications of fNIRS in nonsurgical settings employing scalp probes. ${ }^{29,30}$ Sato et al. ${ }^{14}$ applied fNIRS intraoperatively to study language system corticocortical networks. Our study demonstrates the intraoperative application of fNIRS for mapping eloquent brain regions validated by DCS. Based on a previous design, ${ }^{13}$ our smaller, lighter, and higher spatial resolution probe was designed to map brain activity intraoperatively by directly placing on the brain surface to detect near-infrared light signals permeating through the brain cortex due to appropriate signal depth.
For most patients, we observed $\mathrm{O}_{2} \mathrm{Hb}, \mathrm{HHb}$, and $\mathrm{tHb}$ alterations in real-time during continuous mapping of eloquent brain regions. Our results also showed a positive correlation between positive fNIRS results and patients' blood $\mathrm{Hb}$ concentration and MBP. In the cerebral cortex transitioning from the resting to the active state, the concentration of $\mathrm{O}_{2} \mathrm{Hb}$ in local surface blood vessels increases due to arteriolar vasodilation with a concomitant decrease in $\mathrm{HHb}$ concentration. This is due to neurovascular coupling, a mechanism causing local arteriolar vasodilation to increase local cerebral blood flow and blood volume in response to neurological activation. The fNIRS detector spectrally interrogates this modulation in local brain $\mathrm{Hb}$ concentrations based on the location of the measuring point and accurate positioning of the probes, which are carefully adjusted to obtain good lateral and depth spatial resolution. ${ }^{12,19}$ The large amount of oxygen transported to these activated brain regions generally exceeds the local tissue's rate of oxygen consumption, leading to an increased local concentration of $\mathrm{O}_{2} \mathrm{Hb}$. The flow of blood also flushes away $\mathrm{HHb}$, which could explain its decline in these regions.

In some instances, there was an initial slight decrease in $\mathrm{O}_{2} \mathrm{Hb}$ concentration and a concomitant increase in $\mathrm{HHb}$. As $\mathrm{tHb}$ is a sum of $\mathrm{O}_{2} \mathrm{Hb}$ and $\mathrm{HHb}$ concentrations, its alterations could reflect the extent of oxygenation and perfusion of activated brain regions in response to tasks. If explored, this can offer insight into the vascularity of different brain regions. The spiky nature of the language curves could be a reflection of neural processes underlying thinking and word generation, which are more complex than simple motor tasks.

To illustrate our findings clinically, we presented two cases of successful and one case of failed brain mapping using fNIRS. The first case demonstrates the typical response to cerebral activation seen in most patients, which occured during continuous mapping of task periods (Fig. 3). During language- and motor-related tasks, there was a large rise in $\mathrm{O}_{2} \mathrm{Hb}$ concentration in the probed region, which is explained by local arteriolar blood flow in response to cortical activation, as described previously. However, in this group of patients, the $\mathrm{HHb}$ curve remained fairly dissociated with changes in brain activation. This could be due to decreased sensitivity of the probe to $\mathrm{HHb}$ changes in these patients or a decreased temporal resolution for detecting rapidly fluctuating $\mathrm{HHb}$ changes. The persistence of elevated $\mathrm{O}_{2} \mathrm{Hb}$ levels during the rest period could be attributed to sustained arteriolar vasodilation in activated brain regions. The second case illustrates fNIRS response to brain activation, where the $\mathrm{O}_{2} \mathrm{Hb}$ curve decreases in response to tasks while $\mathrm{HHb}$ rises. This was clearly seen in number counting tasks, speaking to the high metabolic rate of these brain regions leading to rapid oxygen consumption and deoxygenation. Our third illustrated case represents an instance of inaccurate fNIRS detection of brain function. This may be due to some underlying histopathological discrepancy that altered the path through which photons tracked in the brain tissue leading to disrupted readings on the detector. The aberrant result could also be a reflection of previous DCS causing these regions to become activated and irresponsive to subsequent activation. Another possible explanation could be the limited resolution of the detector making it insensitive to weaker signals. In any case, further studies on larger cohorts are required to develop standardized criteria for fNIRS brain mapping.

Over the years, fNIRS has gathered increasing attention as a potential and cheaper approach to complement present routine 

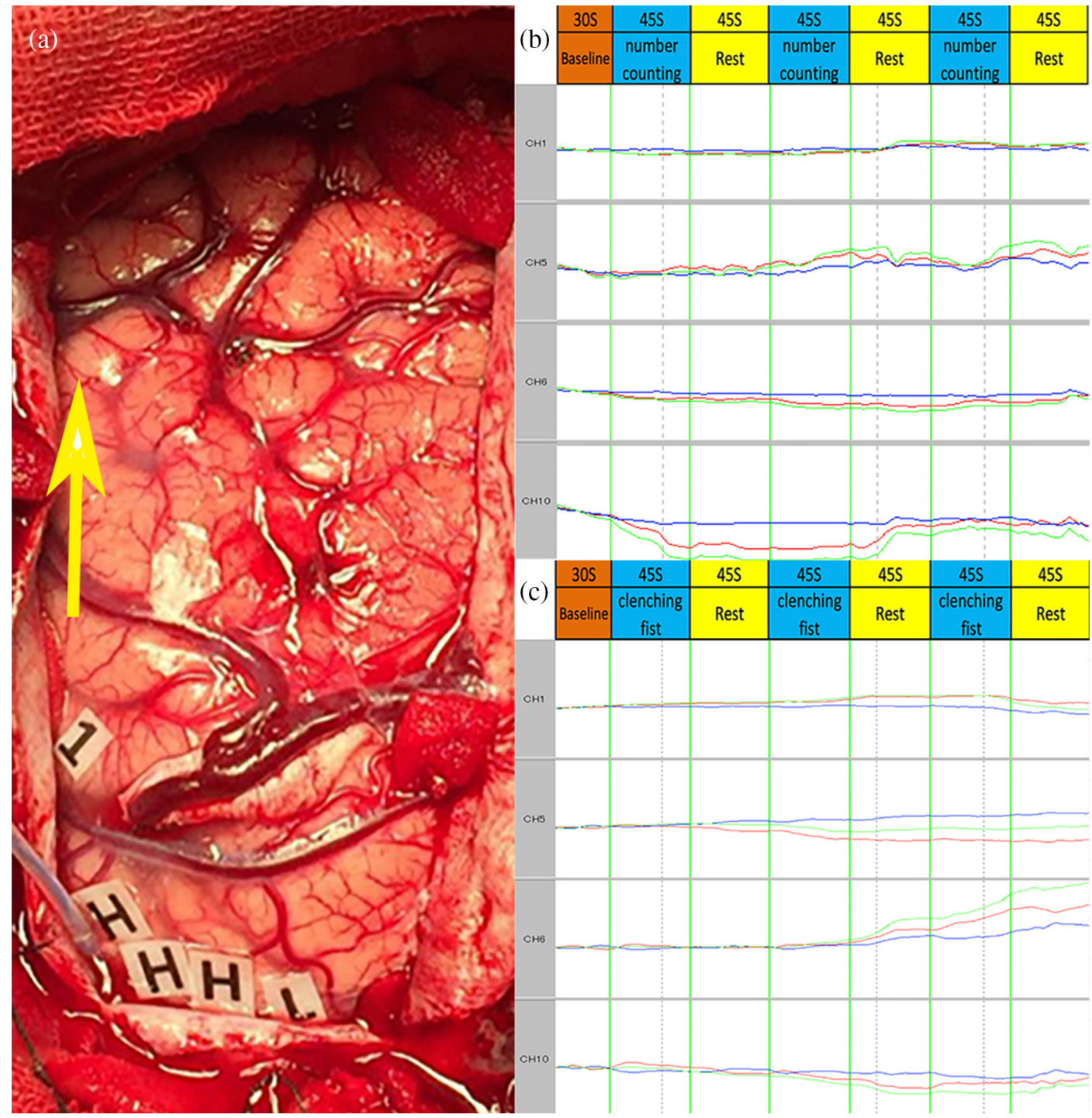

Fig. 5 Illustrated case 3. Following (a) awake craniotomy and DCS (arrow points in the anterior direction); (b) and (c) aside from a few exceptions, the $\mathrm{O}_{2} \mathrm{Hb}$ (red), $\mathrm{HHb}$ (blue), and $\mathrm{tHb}$ (green) curves all trended close to baseline and did not respond to brain task activation.

imaging techniques. A recent study by our group demonstrated the efficacy of fMRI for intraoperative real-time motor mapping. ${ }^{31}$ Compared with those subjected to DCS, the sensitivity and specificity of intraoperative resting-state fMRI in localizing the motor area were $61.7 \%$ and $93.7 \%$, respectively. However, as our continuous mapping results in the present study demonstrate, fNIRS permits real-time intraoperative mapping of both language and motor regions, and has higher temporal resolution than fMRI and positron emission tomography. Its spatial resolution also exceeds that of magnetoencephalography and EEG. ${ }^{25}$ Moreover, it is cheap, mobile, magnetically compatible, easy to operate, and robust to subject motion permitting measurements of prolonged duration under fewer physical restrictions and measurements in infants and incompetent individuals. ${ }^{18}$ Given its portability and safety profile, fNIRS technology makes an interesting prospect to be used in less developed regions and hospitals lacking expensive modern imaging techniques.

fNIRS has the potential to be implemented in routine clinical practice in the not-so-distant future and transform brain mapping from traditional, indirect, invasive, structure-based, and low- resolution methods to a direct, less-invasive, function-based, and higher-resolution approach. However, there are some limitations associated with fNIRS. In addition to limitations of spatial resolution, the depth sensitivity of most fNIRS equipment is $\sim 25$ to $30 \mathrm{~mm}$. This limits investigations to only the outer parts of the cortex, restricting fNIRS functional studies targeting deep brain regions. Also, the fNIRS probe is bulky with only four local sensors. In addition to further validation by DCS, developing a membrane-like probe with more detectors can permit simultaneous analysis of multiple neural networks. Also, our analysis was unable to determine the specificity of fNIRS, given the small size of the probes, which would require a significant extension of surgery time. It is possible that other regions that did not stimulate positively by DCS could have exhibited similar peaks during tasks. These limitations need to be addressed in future studies to expand the applications of fNIRS.

\section{Conclusion}

fNIRS is capable of intraoperative real-time mapping of functional cortical regions, but its accuracy needs development. 
Providing the device develops accuracy and efficiency, it has the potential to supplement, or even replace, DCS.

\section{Disclosures}

The authors have no conflicts of interest to disclose.

\section{References}

1. D. J. Edwards et al., "Raised corticomotor excitability of M1 forearm area following anodal tDCS is sustained during robotic wrist therapy in chronic stroke," Restor. Neurol. Neurosci. 27(3), 199-207 (2009).

2. R. Lindenberg et al., "Bihemispheric brain stimulation facilitates motor recovery in chronic stroke patients," Neurology 75(24), 2176-2184 (2010).

3. D. M. Blumberger et al., "A randomized double-blind sham-controlled study of transcranial direct current stimulation for treatment-resistant major depression," Front. Psychiatry 3, 74 (2012).

4. M. Zimerman et al., "Modulation of training by single-session transcranial direct current stimulation to the intact motor cortex enhances motor skill acquisition of the paretic hand," Stroke 43(8), 2185-2191 (2012).

5. D. Wang, Y. Wu, and J. Xia, "Review on photoacoustic imaging of the brain using nanoprobes," Neurophotonics 3(1), 010901 (2016).

6. F. Irani et al., "Functional near infrared spectroscopy (fNIRS): an emerging neuroimaging technology with important applications for the study of brain disorders," Clin. Neuropsychol. 21(1), 9-37 (2007).

7. S. Ulkatan et al., "Incidence of intraoperative seizures during motor evoked potential monitoring in a large cohort of patients undergoing different surgical procedures," J. Neurosurg. 126(4), 1296-1302 (2017).

8. R. C. Oldfield, "The assessment and analysis of handedness: the Edinburgh inventory," Neuropsychologia 9(1), 97-113 (1971).

9. D. A. Boas et al., "The accuracy of near infrared spectroscopy and imaging during focal changes in cerebral hemodynamics," Neuroimage 13(1), 76-90 (2001).

10. X. Xiao et al., "Transcranial brain atlas," Sci. Adv. 4(9), eaar6904 (2018).

11. C. A. Giller et al., "A stereotactic near-infrared probe for localization during functional neurosurgical procedures: further experience," $J$. Neurosurg. 110(2), 263-273 (2009).

12. A. Villringer, "Understanding functional neuroimaging methods based on neurovascular coupling," in Optical Imaging of Brain Function and Metabolism 2, A. Villringer and U. Dirnag1, Eds., Vol. 413, pp. 177193, Springer, Boston, Massachusetts (1997).

13. M. Fukuda et al., "Cortico-cortical activity between the primary and supplementary motor cortex: an intraoperative near-infrared spectroscopy study," Surg. Neurol. Int. 6, 44 (2015).

14. K. Sato et al., "Cortico-cortical evoked hemodynamic responses in human language systems using intraoperative near-infrared spectroscopy during direct cortical stimulation," Neurosci. Lett. 630, 136-140 (2016).

15. C. Hirth et al., "Non-invasive functional mapping of the human motor cortex using near-infrared spectroscopy," Neuroreport 7(12), 19771981 (1996).

16. Y. Hoshi and M. Tamura, "Near-infrared optical detection of sequential brain activation in the prefrontal cortex during mental tasks," Neuroimage 5(4), 292-297 (1997).

17. G. Taga et al., "Brain imaging in awake infants by near-infrared optical topography," Proc. Natl. Acad. Sci. U. S. A. 100(19), 10722-10727 (2003).

18. T. Wilcox et al., "Using near-infrared spectroscopy to assess neural activation during object processing in infants," J. Biomed. Opt. 10(1), 011010 (2005).

19. S. C. Bunce et al., "Functional near-infrared spectroscopy," IEEE Eng. Med. Biol. Mag. 25(4), 54-62 (2006).

20. T. J. Huppert et al., "A temporal comparison of BOLD, ASL, and NIRS hemodynamic responses to motor stimuli in adult humans," Neuroimage 29(2), 368-382 (2006).

21. L. Holper, M. Biallas, and M. Wolf, "Task complexity relates to activation of cortical motor areas during uni- and bimanual performance: a functional NIRS study," Neuroimage 46(4), 1105-1113 (2009).
22. S. P. Koch et al., "High-resolution optical functional mapping of the human somatosensory cortex," Front. Neuroenerg. 2, 12 (2010).

23. A. Antal et al., "Imaging artifacts induced by electrical stimulation during conventional fMRI of the brain," Neuroimage 85(Pt. 3), 1040-1047 (2014).

24. S. Lloyd-Fox et al., "Are you talking to me? Neural activations in 6-month-old infants in response to being addressed during natural interactions," Cortex 70, 35-48 (2015).

25. H. Y. Kim et al., "Application of functional near-infrared spectroscopy to the study of brain function in humans and animal models," Mol. Cells 40(8), 523-532 (2017).

26. F. Wang et al., "Intersession instability in fNIRS-based emotion recognition," IEEE Trans. Neural Syst. Rehabil. Eng. 26(7), 1324-1333 (2018).

27. M. A. Yücel et al., "Functional near infrared spectroscopy: enabling routine functional brain imaging," Curr. Opin. Biomed. Eng. 4, 78-86 (2017).

28. J. Lu et al., "Awake language mapping and 3-Tesla intraoperative MRIguided volumetric resection for gliomas in language areas," J. Clin. Neurosci. 20(9), 1280-1287 (2013).

29. Y. Inoue et al., "Reduced prefrontal hemodynamic response in children with ADHD during the Go/NoGo task: a NIRS study," Neuroreport 23(2), 55-60 (2012).

30. A. Araki et al., "Improved prefrontal activity in AD/HD children treated with atomoxetine: a NIRS study," Brain Dev. 37(1), 76-87 (2015).

31. T. M. Qiu et al., "Real-time motor cortex mapping for the safe resection of glioma: an intraoperative resting-state fMRI study," AJNR Am. J. Neuroradiol. 38(11), 2146-2152 (2017).

Tianming Qiu received his MD and PhD degrees in neurosurgery from Shanghai Medical School, Fudan University, in 2008 and 2014 , respectively. He is an attending physician working in the Department of Neurosurgery, Huashan Hospital, Fudan University, China. His current clinical work focuses on brain tumor surgeries including awake surgeries. His research interests include development of functional near-infrared spectroscopy (fNIRS) and functional magnetic resonance imaging (fMRI).

N. U. Farrukh Hameed received his MBBS from Southeast University in 2015, and his MCh degree from Fudan University in 2018. He is currently a PhD student in the Department of Neurosurgery, Huashan Hospital, Fudan University, China. His research focuses on functional and molecular mapping of brain regions.

Yuerong Peng received her MD degree from Shanghai Medical School Fudan University in 2008. She is an attending physician working in the Department of Anesthesiology, Huashan Hospital, Fudan University, China. Her current clinical work and research focuses on awake anesthesia.

Shuheng Wang received his BS degree in applied mathematics from the University of California, San Diego, in 2018, and his MA degree in statistics and data science from Yale University in 2019. His research interests center on applied statistics and machine learning, including inference on ultra high-dimensional data, regression, and classification algorithms, survival analysis, statistical fMRI analysis, and model selection methods.

Jinsong Wu received his $M D$ and $\mathrm{PhD}$ degrees in neurosurgery from Shanghai Medical School, Fudan University, in 1995 and 2000, respectively. Currently, he is a professor working in the Department of Neurosurgery, Huashan Hospital, Fudan University, China. He is also a vice director of the Glioma Surgery Division and the director of Brain Function Laboratory, Neurosurgical Institute of Fudan University. His research interests include development of advanced technologies for brain mapping.

Liangfu Zhou received his MD degree in neurosurgery from Shanghai First Medical School (now Shanghai Medical School, Fudan University) in 1965. He is the chief of the Department of Neurosurgery, Huashan Hospital, Fudan University, China. He is an academician of the Chinese Academy of Engineering. He has long been working in the fields of neurosurgery and neuroscience. 